\title{
The Placebo Effect: Advances from Different Methodological Approaches
}

\author{
Karin Meissner, ${ }^{1,2 *}$ Ulrike Bingel, ${ }^{3 \star}$ Luana Colloca, ${ }^{4,5 *}$ Tor D. Wager, ${ }^{6 *}$ Alison Watson, ${ }^{7 \star}$ and Magne Arve Flaten ${ }^{8 *}$ \\ ${ }^{1}$ Institute of Medical Psychology, Ludwig-Maximilians-University of Munich, D-80336 Munich, Germany, ${ }^{2}$ Institute of General Practice, Technical \\ University of Munich, D-81776 Munich, Germany, ${ }^{3}$ NeuroImage Nord, Department of Neurology, University Medical Center Hamburg-Eppendorf, D-20246 \\ Hamburg, Germany, ${ }^{4}$ National Center for Complementary and Alternative Medicine and ${ }^{5}$ Department of Bioethics, National Institutes of Health, Bethesda, \\ Maryland 20892-1156, ${ }^{6}$ Department of Psychology, University of Colorado, Boulder, Colorado 80309-0345, ${ }^{7}$ Human Pain Research Group, University of \\ Manchester, Manchester MI3 9PT, United Kingdom, and ${ }^{8}$ Department of Psychology, University of Tromsø, 9037 Tromsø, Norway
}

There is accumulating evidence from different methodological approaches that the placebo effect is a neurobiological phenomenon. Behavioral, psychophysiological, and neuroimaging results have largely contributed to accepting the placebo response as real. A major aspect of recent and future advances in placebo research is to demonstrate linkages between behavior, brain, and bodily responses. This article provides an overview of the processes involved in the formation of placebo responses by combining research findings from behavioral, psychophysiological, and neuroimaging methods. The integration of these different methodological approaches is a key objective, motivating our scientific pursuits toward a placebo research that can inform and guide important future scientific knowledge.

\section{Introduction}

Placebo treatments have been shown to influence reported pain experiences in dozens of well controlled studies. In many domains, these findings would be viewed as incontrovertible evidence that placebos influence pain.

However, many lay people, medical professionals, and scientists alike appear uncomfortable with the idea that placebo effects, by leading people to believe they suffer from less pain, can cause them to actually experience the same feelings and adverse consequences as if they had received an effective treatment. If patients on placebo say they feel less pain, then they do, and that is what counts for the patient. However, this position is not very satisfying, due to a potentially fundamental error in reasoning, i.e., the assumption that patients' reports are straightforward, accurate accounts of an internal reality (pain and pain-related processes). But not all patient reports are equally created. There are many reasons that patients might report less pain, some of which have more to do with the desire to be self-consistent, "correct," liked by their caregiver, and other factors independent of the pain itself. It has been suggested that placebo effects are created by these and similar cognitive biases (Clark, 1969; Feather et al., 1972; Allan and Siegel, 2002). For example, according to sig-

\footnotetext{
Received Aug. 9, 2011; revised Sept. 11, 2011; accepted Sept. 13, 2011.

This work has been funded in part by generous support from intramural NCCAM (L.C.), International Association for the Study of Pain (IASP) and European Federation of IASP Chapters research grants (L.C.), The Bial Foundation Grant 186/10 (M.A.F.), NIMH Grant R01MH076136 (T.D.W.), NIDA Grant 1RC1DA028608 (T.D.W.), the German Federal Ministry of Education and Research (01GQ0808) (U.B.), and the German Research Foundation (F0-1328) (U.B.).

${ }^{*}$ All the authors contributed equally to this work.

Correspondence should be addressed to either of the following: Dr. Magne Arve Flaten, Department of Psychology, Faculty of Health Sciences, University of Tromsø, 9037 Tromsø, Norway. E-mail: magne.flaten@uit.no; or Dr. Luana Colloca, National Center for Complementary and Alternative Medicine, National Institutes of Health, Building 10, Room 1C118, Bethesda, MD 20892-1156. E-mail: luana.colloca@nih.gov.

DOI:10.1523/JNEUROSCI.4099-11.2011

Copyright $\odot 2011$ the authors $\quad 0270-6474 / 11 / 3116117-08 \$ 15.00 / 0$
}

nal detection theory, the expectation of a treatment creates uncertainty about the sensory information of pain, and the placebo response is a case of perceptual error (Allan and Siegel, 2002). Indeed, there is some evidence that the tendency to use prior expectation when making perceptual judgments, instead of independently evaluating available perceptual information, is a general cognitive style that is positively associated with placebo analgesic responses (Morton et al., 2010a).

One way to understand whether the placebo effect is 'real' is to define particular physiological and functional outcomes that are in themselves meaningful. The combination of behavioral, neuroimaging, and psychophysiological studies have been particularly useful in this regard, particularly in the field of pain as they provide information about the brain and bodily changes that accompany placebo responses. A major aspect of recent and future advances in the placebo research is to demonstrate and to emphasize linkages between behavior, brain, and bodily responses. We seek to integrate the different methodological approaches that are being used to augment current scientific knowledge. Thus, we summarize the psychological mechanisms underlying the formation of placebo responses, the modulator role of negative emotion in placebo analgesia and nocebo hyperalgesia, the activation of endogenous pain modulator networks during placebo analgesia, the causal relationship between placebo analgesic and functional magnetic resonance imaging (fMRI) responses, and finally, the placebo-induced peripheral effects.

Psychological mechanisms of the placebo effect: contribution of verbal suggestions, conditioning, and social observation Classical conditioning and expectations are generally accepted to be the most prominent mechanisms of the placebo effect. If the goal is to provide a comprehensive knowledge of the placebo effect, any rigid distinction between conditioning and expectations might be flawed. Remarkably, sophisticated pain models 


\section{Psychosocial signals}

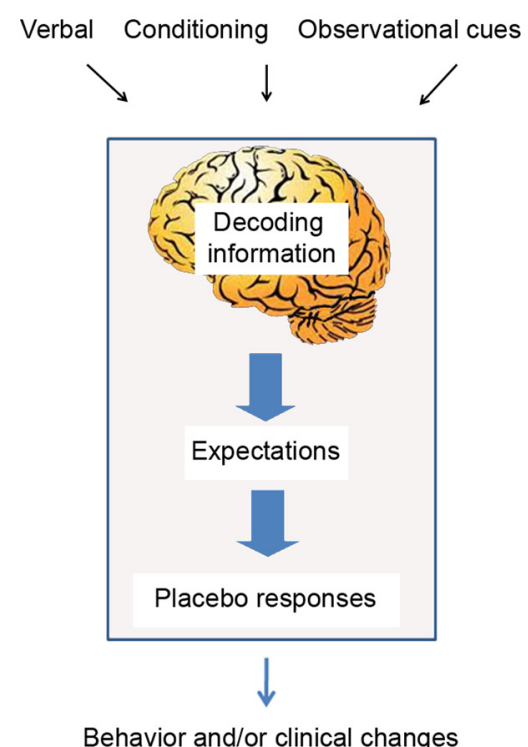

Figure 1. The scheme shows how psychosocial signals including conditioning, verbal and observational cues are detected by the brain, interpreted, and translated into neural inputs crucial to form expectations and placebo responses resulting in behavior and clinical changes (adapted from Colloca and Miller, 2011a).

and brain imaging techniques being used in placebo research are leading to a better understanding of the basic mechanisms underlying the formation of placebo responses. Verbal, conditioned, and observational cues can create strong expectations that influence the brain placebo response and lead to the release of endogenous opioids and dopamine (Fig. 1).

Ostensibly, most of our current knowledge on placebo mechanisms has been gained by investigation of the placebo effect in the domain of pain (Colloca and Benedetti, 2005; Tracey, 2010), and a classic example is that of a subject or a patient who experiences pain relief after being presented with a placebo described as an analgesic medication. The patient may experience analgesia simply by virtue of this anticipation of relief. The interpretation of verbal suggestions in communication between therapist and patient has also been shown to relieve anxiety, which often exacerbates suffering from illness (Colloca and Benedetti, 2007; Aslaksen et al., 2011). Furthermore, the association of specific cues to the experience of the treatment effect induced by pharmacological or biological manipulations significantly alters behavior and clinical outcomes. A conditioned stimulus (CS), such as a pill's color, conveys information about the unconditioned stimulus (US), the effect of the drug on a symptom like pain (Rescorla, 1988). After learning the relation between the CS and the US, presentation of the CS leads to an anticipation of the US that may result in a placebo conditioned response such as a reduction of the pain experience (Colloca et al., 2008a,b). In short, the conditioned placebo effect leads the individual to experience a beneficial outcome. Some recent studies adopting a pharmacological conditioning with drugs such as morphine (Amanzio and Benedetti, 1999; Benedetti et al., 2007), immunosuppressor cyclosporin A (Goebel et al., 2002; Pacheco-López et al., 2009), dopamine-agonist apomorphine (Benedetti et al., 2004), benzodiazepine receptor agonist midazolam, and antagonist flumazenil (Petrovic et al., 2002) found that the conditioned placebo responses mimic drug effects, thus providing evidence that an important factor in eliciting bodily placebo responses is the previous exposure to effective treatment. Conditioned placebo analgesic responses appear to be sensitively shaped not only by prior exposure to treatment effectiveness (Colloca and Benedetti, 2006; Morton et al., 2010a,b), but also by the number of learning trials (Colloca and Benedetti, 2006, 2009; Colloca et al., 2008a,b, 2010; Colloca and Miller, 2011a).

The experience of effectiveness also affects pain clinical outcomes in patients with neuropathic pain (André-Obadia et al., 2011). Conditioned responses to drugs as USs are not only compensatory or antagonistic physiological responses (Siegel, 2008). When drugs restore homeostasis, e.g., reduction of pain, an agonistic placebo conditioned response can be observed (Flaten, 2009).

People can also form placebo responses by observing others in a social context without any deliberate reinforcement to the observer. Colloca and Benedetti (2009) investigated the role of social observation in placebo analgesia in healthy subjects who learned by observing a demonstrator who simulated an analgesic benefit. Substantial placebo analgesic responses were found following observation of the demonstrator, suggesting that the information drawn from the observation of another person may establish a self-projection into the future outcome. These effects exhibited a magnitude that was similar to those induced by directly experiencing the benefit through a conditioning procedure in which subjects underwent first-hand experience of benefit. Importantly, higher empathy yielded stronger placebo responses, supporting a link between the ability to modify behaviors following social observational learning and empathy. Beyond empathy, some other dispositional traits such as altruism (Mackenbach, 2005) and optimism (Geers et al., 2005, 2010; Morton et al., $2010 \mathrm{~b}$ ) seem to play a role, indicating that emotions and personality traits modulate expectations and placebo-induced behavioral changes.

Research has also shown that expectations can modify the response to active analgesic treatments and interventional procedures both positively and negatively. For example, patients who underwent a thoracotomy and received morphine for postoperative pain showed significantly better pain relief when they were told that they would receive morphine and their pain would get better soon. However, patients given similar therapeutic dosages by a computer-controlled infusion pump and who were not told when treatment began had a significant reduction in analgesic drug effects (Colloca et al., 2004). In another study, when healthy subjects were told that a drug infusion of a $\mu$-opioid agonist, remifentanil, was going to be started, the analgesic effect was doubled as compared to patients who received no verbal suggestions about the medication. Moreover, informing subjects that the remifentanil was stopped when actually the subjects continued to receive the drug led to a reduced analgesic effect and brain related-changes. This observation suggests that expectations can strongly interfere with the response to painkillers, even reversing their potential analgesic properties (Bingel et al., 2011). These findings indicate that understanding the mechanisms mediating expectancies and placebo responses is a form of neuroscience clinically relevant to symptom management.

\section{Is placebo analgesia mediated via reduced negative emotions?}

Recent research has suggested that placebo analgesia may be mediated via reduced negative emotions (Flaten et al., 2011). Pain increases negative emotions like nervousness and anxiety, and negative emotions increase pain, whereas a reduction in negative emotions has been shown to reduce pain (Rhudy et al., 2008). 
When treatment for pain is administrated, the patient expects the unpleasant symptoms to decrease. Thus, it could be hypothesized that the expectation of having received effective treatment reduces nervousness and anxiety, which in turn reduces unpleasant symptoms like pain (Aslaksen and Flaten, 2008; Aslaksen et al., 2011). This seemingly simple hypothesis has been difficult to investigate, since stress levels are generally low in healthy volunteers, and effects of placebos on stress are small at the group level in these participants (Flaten et al., 2006). However, at the individual level, the negative effects of stress on pain and placebo analgesia have been demonstrated in subjects with higher stress levels, namely, in subjects that were fearful of pain. Lyby et al. (2010) induced heat pain in healthy volunteers, and found that higher scores in fear of pain were associated with higher stress levels during anticipation of pain, and during painful stimulation. Subjects high in fear of pain showed smaller or no placebo analgesic responses, confirming that stress reduces placebo analgesia. Conversely, individuals who scored highly in dispositional optimism tended to show a drop in state anxiety after their first exposure to a placebo. This subsequently led to reduced anxiety at the second exposure with a corresponding placebo response, which seemed to last for at least 6 weeks (Morton et al., 2010a,b).

Aslaksen et al. (2011) observed that placebos-administered with information that they were powerful painkillers-reduced stress in the absence of pain and before the placebo analgesic response to painful stimulation was observed. This finding suggests that the reduced stress after administration of a placebo cannot be explained by reduced pain that in turn reduces stress. Rather, the reduced stress predicted the placebo analgesic response, suggesting that the reduced stress explained the placebo response. However, these findings were made in males only. In females, there was no placebo response, and there was no effect of placebos on stress. The absence of placebo responses in females was explained by increased stress levels that were not modulated by placebo administration (Lyby et al., 2011). Remarkably, eventrelated potentials (ERPs) to painful stimulation were also reduced in the placebo condition compared to a natural history condition where pain was applied, but no pills and no information were administrated. As for pain report, smaller ERPs after placebo was only observed in males, and reduced reported stress predicted smaller ERPs to painful stimulation. ERP reduction was observed in the N2 and P2 peaks that register activity from the cingulate gyrus and the insular areas (Garcia-Larrea et al., 2003). These cortices are central for emotional and pain processing and for placebo responding (Wager et al., 2004).

If placebo responses are due to reductions in stress levels, then the nocebo response, often considered the opposite of placebo responses, could be due to increases in stress levels. Nocebo responses are often observed as increases in pain after a substance or procedure has been administrated with information that it will increase pain. This induces nervousness and anxiety (Flaten et al., 1999); e.g., cortisol has been shown to increase after this information (Johansen et al., 2003). Pharmacological blockade of anxiety abolishes the nocebo response (Benedetti et al., 2006a). Thus, placebo and nocebo responses may be seen as different ends of a continuum from very little negative emotions like nervousness and tension to extreme negative emotions: placebo effects are observed in subjects where the inert placebo induces relaxation, whereas nocebo effects may be seen when nervousness and anxiety are induced.

Interesting studies have also shown that the interaction between expectations and desire of pain relief contributes to placebo analgesia in a patient population suffering from chronic pain due to irritable bowel syndrome (IBS). Patients with IBS were exposed to rectal balloon distension under no treatment, rectal placebo, and rectal lidocaine to investigate whether expectations and desire for pain relief impact the therapeutic responses to active and placebo treatments. By assessing expectations and desires of pain relief before and after each treatment, the authors found that $77 \%$ of the variance in placebo analgesia and $81 \%$ of the variance in analgesia induced by lidocaine was related to individual beliefs (Vase et al., 2003). Patients with IBS were also investigated under no treatment, rectal placebo, and rectal lidocaine in another study aimed at exploring the dynamic impact of expectations, desires for pain relief, and anxiety on the formation of analgesic responses (Vase et al., 2005). There was a trend of increased analgesia over the entire session of rectal distension (40 $\mathrm{min})$. Expectations, pain relief desires, and anxiety accounted for a substantial variation in the response to placebo and lidocaine treatment, suggesting that these psychological variables may contribute to the experience of analgesia. These results have been confirmed by a brain imaging study wherein fMRI was used to detect the association among psychological variables, brain responses and pain reports. The modulation of brain areas involved in the processing of afferent and visceral pain was dynamically related to patients' expectations (Price et al., 2007). Based on these and other studies, we can assume that expectations and desires are important determinants of the brain and bodily responses to active treatments and placebos, as they induce emotions that modulate pain experience.

\section{Neuroimaging studies of placebo analgesia}

The current state of the art in this field is summarized in the following by focusing on two kinds of questions: First, do placebo treatments influence the "pain processing network" - the set of regions most closely associated with pain experience-and, if so, which of its components? An affirmative answer would suggest that aspects of pain experience are influenced in ways that may be functionally and physiologically important. Second, what kinds of changes in brain processes may mediate effects in pain? Information about these processes could yield plausible mechanisms of placebo responses. We briefly address each question in turn, presenting the current consensus in the field and identifying several unresolved issues.

Since the pioneering positron emission tomography study on placebo analgesia by Petrovic et al. (2002) first revealed a shared neural network of the rostral anterior cingulate cortex (rACC) and the brainstem underlying both opioid and placebo analgesia, the relevance of this network for placebo analgesia has been substantiated by several neuroimaging studies using various procedures to induce placebo analgesia (including placebo analgesic creams, sham acupuncture, and others) (Wager et al., 2004, 2007; Zubieta et al., 2005; Bingel et al., 2006; Kong et al., 2006; Eippert et al., 2009b). These studies showed that placebo analgesia involves the activation of cingulo-frontal brain regions together with subcortical structures such as the midbrain periaqueductal gray (PAG), hypothalamus, and amygdala. Connectivity analyses further revealed that the behavioral placebo analgesic effect depends on an enhanced functional coupling of the rACC with brainstem areas such as the PAG (Amanzio and Benedetti, 1999; Wager et al., 2004, 2007; Eippert et al., 2009b). These studies support the notion that the top down activation of endogenous analgesic activity via the descending modulatory system represents an integral part of the mechanisms of placebo analgesia. The opioidergic nature of this pain-modulating system has been corroborated by both pharmacological studies using the opioid an- 


\section{A Decreases in pain-related responses}

\section{B Placebo-related activity increases}
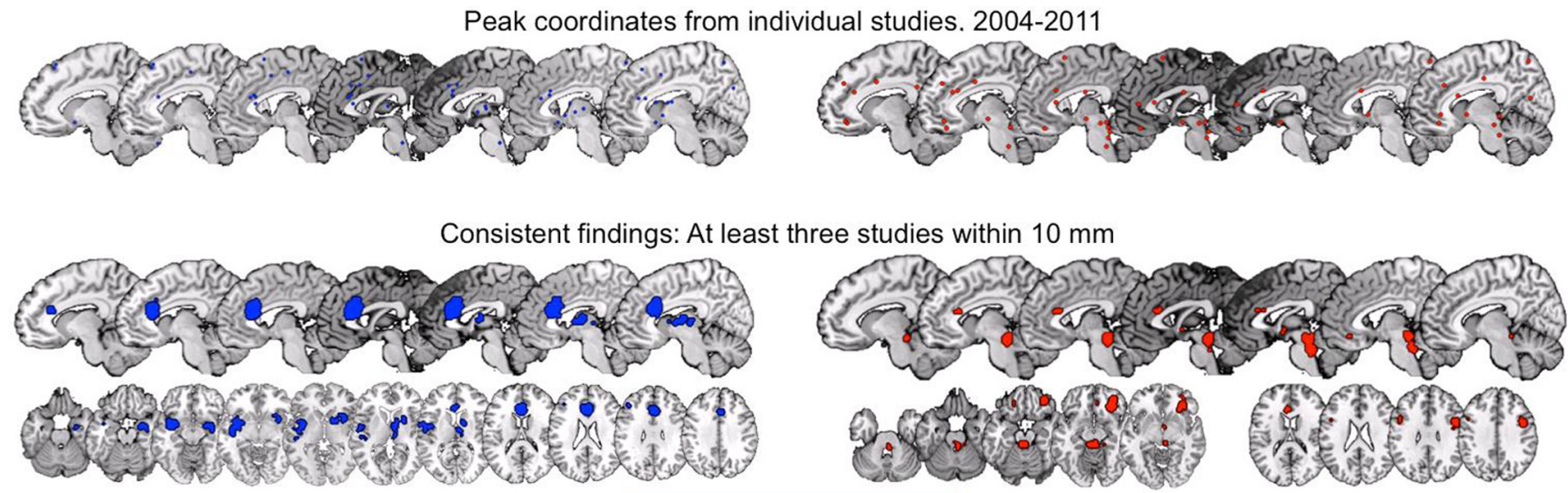

Activity decreases

Activity increases

Figure 2. Consistent placebo-related neuroimaging findings from 2004 to 2011. A complete list of studies can be found in Wager and Fields (2011). $A$, Placebo-related decreases during pain, or regions in which placebo-related decreases were correlated with placebo analgesia. $\boldsymbol{B}$, Placebo-related increases in neuroimaging activity during anticipation or pain, or regions in which placebo-related increases correlated with placebo analgesia. The top rows show individual peak coordinates (for more details, see Wager and Fields, 2011), and the bottom rows show areas activated in at least three separate maps. Note that increases and decreases can be found in the same brain regions, if, e.g., there are consistent increases during anticipation of pain and decreases during pain experience. This is the case with the rostral dorsal cingulate region shown in both maps.

tagonist naloxone and in vivo receptor binding approaches (Levine et al., 1978; Amanzio and Benedetti, 1999; Zubieta et al., 2005; Eippert et al., 2009b).

Another important brain area for placebo analgesia is the prefrontal cortex. Activity in the dorsolateral prefrontal cortex was found in the period preceding noxious stimulation, which correlated with activity in the PAG and the subsequent placebo analgesic response (Wager et al., 2004; Eippert et al., 2009b; Watson et al., 2009; Lui et al., 2010; Wager et al., 2011; Wager and Fields, 2011). Both temporary functional lesions of the prefrontal cortex by repetitive transcranial magnetic stimulation (Krummenacher et al., 2010) as well as the degeneration and disconnection of the frontal lobes in Alzheimer's disease (Benedetti et al., 2006b) are associated with a reduction or complete loss of verbally induced placebo analgesic responses.

The majority of neuroimaging studies of placebo analgesia indicate that the reduced pain ratings during placebo analgesia are paralleled by decreased activity in the classical pain processing areas including the thalamus, insula, and the somatosensory cortex (Petrovic et al., 2002; Wager et al., 2004; Price et al., 2007; Lyby et al., 2011; Wager and Fields, 2011). Recent evidence from spinal cord fMRI, which only recently became technically feasible in both humans and animals, revealed that pain-related activity in the ipsilateral dorsal horn, corresponding to painful stimulation, is substantially reduced under placebo (Eippert et al., 2009a). These results provided direct evidence for spinal inhibition as one mechanism of placebo analgesia and highlight the fact that psychological factors such as expectancy can act on the earliest stages of pain processing in the CNS. Together these studies support the notion that altered pain experience during placebo analgesia, at least in part, results from active inhibition of nociceptive activity and not simply from report bias.

Areas with consistent placebo-related reductions and increases across studies (with peak coordinates within $10 \mathrm{~mm}$ in at least three studies) are shown in Figure 2. A number of regions that do not have a clear relationship with pain are engaged by placebo treatments, including the lateral prefrontal, orbitofrontal (OFC), and anterior insular cortices and the PAG or surrounding area (for review, see Wager and Fields, 2011). Some areas, including opioid-rich PAG and its targets in OFC and medial prefrontal cortex and subcortical structures (e.g., nucleus accumbens and amygdala), also show evidence for increased opioid system activation with placebo (Wager et al., 2007; Scott et al., 2008), which fits with previous findings showing opioid-dependent placebo effects (Levine et al., 1979; Amanzio and Benedetti, 1999; Eippert et al., 2009a).

\section{Is there a causal relationship between placebo analgesic and fMRI responses?}

To show reduced responses in several regions strongly identified with pain processing, including contralateral anterior insula, medial thalamus, and rostral dorsal cingulate (corresponding to Vogt's anterior midcingulate region) (Wager et al., 2004), was important because these areas are associated with nociception and pain in a variety of ways in humans and animals, and are among the regions that most reliably track noxious stimulus intensity in human neuroimaging studies (Coghill et al., 1999; Apkarian et al., 2005; Atlas et al., 2010). If placebos reduce pain experience, especially by blocking early nociceptive processes (Matre et al., 2006; Goffaux et al., 2007), such responses would be predicted.

However, the relationship between activity in these regions and pain processing must be examined in more detail. Even if the set of regions mentioned above responds reliably to pain, it does not follow that activation implies changes in pain (Poldrack, 2006). These regions are heteromodal association areas that respond to multiple types of affective and cognitive events (Bush et al., 2000; Shackman et al., 2011), potentially including basic stimulus salience across modalities (Mouraux et al., 2011). One approach involves establishing more direct links between placebo manipulations, brain responses, and pain reports. A recent study followed this approach and used multilevel mediation to test 
whether activity in "pain-processing" regions formally mediates relationships between experimentally induced expectations about stimulus intensity and trial-by-trial pain reports (Atlas et al., 2010). As in the placebo studies, fMRI responses to a constant-intensity noxious stimulus were reduced when expectations about pain were lower and these reductions mediated reductions in pain report.

Another approach that is currently examined is to examine whether placebo treatment affects the dose-response relationship between noxious stimulus intensity and fMRI activity in these regions. A third, more direct approach would be to use machine learning to define precise patterns of pain that track pain intensity closely, and test for placebo effects on these patterns. Patterns of brain activity are only useful for making inferences about pain (and other psychological phenomena) insofar as they are consistently and specifically activated by pain, so developing pain-specific biomarkers and testing these for placebo effects could be particularly informative. However, this approach has not yet been used, and attempts to directly identify brain biomarkers with these features will be an important direction for future research.

Human brain research on placebo effects is in a very early stage, and there are many unanswered questions. Some of the most basic include understanding of the relationships between (1) cortical and subcortical regions, (2) MRI increases and activation of opioid and dopamine systems in the same areas, and (3) activation of these putative "mechanisms" and placebo analgesia defined by either pain report or pain-related brain activity. Central to these issues is the question of how well activation of these systems can predict the magnitude of placebo analgesia, particularly in individuals who are naive to the experimental procedure. If brain responses could be used to characterize individual differences in placebo analgesia prospectively, it could provide vital information for both understanding the basic science of endogenous regulation and translating neuroimaging research into clinically useful applications. As a step toward addressing this goal, a machine learning regression technique (a family of techniques useful for developing the types of biomarkers suggested above) was used to predict the magnitude of placebo analgesia prospectively, in individuals for whom behavioral placebo report data are not available or are not considered trustworthy (Wager et al., 2011). The approach yields both overall estimates of predictive accuracy (or effect size) normally absent from brain imaging studies and interpretable maps of which brain regions make important contributions to prediction.

Wager et al. (2011) found that anticipatory activity, before pain, was a particularly strong predictor of placebo analgesia (defined by reductions in pain report) in new individuals, explaining $12 \%$ or $44 \%$ of the variance depending on the sample used. Brain responses during pain in "pain processing" areas were significantly but more weakly predictive, and with no consistent directionality across regions: reductions in anterior cingulate and thalamus and increases in anterior insula predicted larger placebo analgesia. In addition, the magnitude of overall reductions across these regions was uncorrelated with the magnitude of placebo analgesia, suggesting that fMRI measures of "pain processing" and self-reported pain may be highly dissociable outcomes. Future research could test the functional implications of verum or placebo treatments that reduce pain-related brain activity, instead of relying purely on pain reports as an outcome measure.

Finally, these results bear on the nature of what processes underlie placebo-related activity in frontal cortical regions. Previously, it was speculated that frontal activity looks remarkably similar in location to regions activated by cognitive control processes (Benedetti et al., 2005), though these regions are activated by many processes, including emotion-regulation processes whose relationship with cognitive control is unclear (Wager et al., 2008). In Wager et al. (2011), the placebo-predictive accuracy of two sets of brain regions was tested: those engaged in cognitive control of working memory (derived from a published metaanalysis of 60 studies) (Wager and Smith, 2003) and those engaged in emotional appraisal (derived from a meta-analysis of 163 studies) (Kober et al., 2008). Surprisingly, activity in only the emotion-related regions predicted placebo analgesia (emotion, relative to working memory, activated more ventral and rostral portions of the lateral prefrontal cortex), which supports the view that emotions play a crucial role for placebo effects on pain.

\section{The placebo effect and the autonomic nervous system: an investigation of peripheral responses}

So far, this review has focused on pain, which is the bestinvestigated condition in terms of placebo mechanisms and thus allows a good insight into the neurobiological basis of placebo effects. Other diseases of the CNS, such as Parkinson's disease, depression, and anxiety, are also affected by placebo interventions, and the underlying mechanisms are increasingly understood (Benedetti et al., 2005). In addition to CNS disorders, placebo interventions also affect peripheral organs, giving further incontrovertible evidence that placebo effects are real neurobiological phenomena.

There is good evidence that peripheral physiological placebo effects can be achieved by classical conditioning procedures: Caffeine-associated stimuli like the smell and taste of coffee have been shown to elicit increased skin conductance responses and startle eyeblink reflexes also in the absence of caffeine (Flaten and Blumenthal, 1999; Mikalsen et al., 2001). Placebo effects on immune function and related diseases could be elicited by immune conditioning, and autonomic pathways are mandatory for this type of placebo effects (Vits et al., 2011).

Most available data from experimental studies confirm the view that verbal suggestions accompanying placebo interventions can also alter inner organ functions, e.g., in the pulmonary, the gastrointestinal, and the cardiovascular system (Kemeny et al., 2007; Meissner et al., 2007; Meissner, 2009, 2011; Meissner and Ziep, 2011; Ronel et al., 2011). For example, a placebo pill, which was described as either stimulating or dampening the stomach, altered not only subjective perceptions of gastric activity, but also the length of gastric contraction periods (Meissner, 2009). Furthermore, a number of studies showed that the inhalation of an alleged bronchoconstrictor drug impaired lung function in asthmatics to a measurable degree, while inhalations of alleged bronchodilators reduced drug-induced bronchoconstriction (Isenberg et al., 1992; Kemeny et al., 2007). However, a recent study failed to demonstrate a direct bronchodilatory placebo effect on lung function in asthma (Wechsler et al., 2011). Several studies examined the acute effects of verbal suggestions on blood pressure; these studies consistently found that hypotensive suggestions lowered systolic blood pressure, whereas verbal suggestions of blood pressure increase increased systolic and diastolic blood pressure as well as heart rate (for review, see Meissner, 2011; Meissner and Ziep, 2011). Finally, one randomized controlled experimental study recently showed the first evidence that verbal suggestions can affect coronary blood flow independently of effects on heart rate and blood pressure (Ronel et al., 2011).

A surprising property of placebo effects on peripheral organ systems elicited by verbal suggestions is their putative specificity: 
three studies that investigated this issue all provided evidence that placebo interventions specifically altered the organ function that was targeted by the placebo intervention, indicating an organspecific response (Butler and Steptoe, 1986; Meissner, 2009; Meissner and Ziep, 2011). These findings are reminiscent of studies in the field of placebo analgesia, where pain reduction was observed only in the placebo-treated part of the body (Montgomery and Kirsch, 1996; Benedetti et al., 1999; Domnick et al., 2011) and could be reversed by the opioid-antagonist naloxone, suggesting a somatotopic activation of endogenous opioid systems (Benedetti et al., 1999). Placebo effects on inner organ functions may likewise be mediated by organotopic activation of visceromotor representations in the insular and medial prefrontal cortices (Meissner, 2000, 2009, 2011; Meissner and Ziep, 2011).

Physical organ functions are under constant inhibitory and excitatory influences from a series of higher brain centers, which range from cortical areas to the lower brainstem region and are summarized as the "central autonomic network" (CAN) (Benarroch, 1993). The CAN includes areas like the cingulate gyrus and the insular cortex, which are also central for placebo responses in pain (Lane and Wager, 2009). The central control of peripheral organs takes place both via the two branches of the autonomic nervous system, namely the sympathetic and the parasympathetic nervous system, and via the hypothalamic neuroendocrine system (Jänig, 2006). So far no study has examined whether placebo effects on peripheral organ functions induced by verbal suggestions are mediated primarily through the autonomic nervous system or through neuroendocrine pathways. However, the time course of verbally induced placebo effects, which occur usually within seconds or minutes after the intervention (Butler and Steptoe, 1986; Meissner, 2009; Meissner and Ziep, 2011; Ronel et al., 2011), argues strongly for a neural mediation, since the neuroendocrine system has significantly longer reaction times (Jänig, 2006). Moreover, the organ specificity of placebo effects on the peripheral organ functions speaks against neuroendocrine mechanisms, whereas it is consistent with the high functional specificity of autonomic nervous system organization (Morrison, 2001; Jänig, 2006). It will be a challenge for future placebo research to elucidate the central mechanisms that mediate placebo effects on peripheral organ functions.

\section{Summary}

In conclusion, we believe that investigating the formation of behavioral and biological changes due to placebos deserves future efforts, as the placebo effect is a "real" neurobiological phenomenon that has important implications for clinical neuroscience research and medical care. Such contexts include a host of cues that convey information having the potential to produce therapeutic effects, and bodily and behavior changes (Colloca and Miller, 2011b). It is important to note that placebo responses are not limited to "inert" placebo interventions. Indeed, proven effective treatments also function as cues, in addition to producing therapeutic effects based on their inherent pharmacological or physiological characteristics, and may be administered in a clinical encounter, with the potential to generate placebo responses that enhance the treatment's therapeutic benefit (Colloca et al., 2004; Bingel et al., 2011; Bjørkedal and Flaten, 2011). The realm and mechanisms of the placebo phenomenon might prompt a determinant shift in the design and process of clinical trials and practices with significant ethical implications upon patient-doctor relationships.

\section{References}

Allan LG, Siegel S (2002) A signal detection theory analysis of the placebo effect. Eval Health Prof 25:410-420.

Amanzio M, Benedetti F (1999) Neuropharmacological dissection of placebo analgesia: expectation-activated opioid systems versus conditioning-activated specific subsystems. J Neurosci 19:484-494.

André-Obadia N, Magnin M, Garcia-Larrea L (2011) On the importance of placebo timing in rTMS studies for pain relief. Pain 152:1233-1237.

Apkarian AV, Bushnell MC, Treede RD, Zubieta JK (2005) Human brain mechanisms of pain perception and regulation in health and disease. Eur J Pain 9:463-484.

Aslaksen PM, Flaten MA (2008) The roles of physiological and subjective stress in the effectiveness of a placebo on experimentally induced pain. Psychosom Med 70:811-818.

Aslaksen PM, Bystad M, Vambheim SM, Flaten MA (2011) Gender differences in placebo analgesia: event-related potentials and emotional modulation. Psychosom Med 73:193-199.

Atlas LY, Bolger N, Lindquist MA, Wager TD (2010) Brain mediators of predictive cue effects on perceived pain. J Neurosci 30:12964-12977.

Benarroch EE (1993) The central autonomic network: functional organization, dysfunction, and perspective. Mayo Clin Proc 68:988-1001.

Benedetti F, Arduino C, Amanzio M (1999) Somatotopic activation of opioid systems by target-directed expectations of analgesia. J Neurosci 19:3639-3648.

Benedetti F, Colloca L, Torre E, Lanotte M, Melcarne A, Pesare M, Bergamasco B, Lopiano L (2004) Placebo-responsive Parkinson patients show decreased activity in single neurons of subthalamic nucleus. Nat Neurosci 7:587-588.

Benedetti F, Mayberg HS, Wager TD, Stohler CS, Zubieta JK (2005) Neurobiological mechanisms of the placebo effect. J Neurosci 25:10390-10402.

Benedetti F, Amanzio M, Vighetti S, Asteggiano G (2006a) The biochemical and neuroendocrine bases of the hyperalgesic nocebo effect. J Neurosci 26:12014-12022.

Benedetti F, Arduino C, Costa S, Vighetti S, Tarenzi L, Rainero I, Asteggiano G (2006b) Loss of expectation-related mechanisms in Alzheimer's disease makes analgesic therapies less effective. Pain 121:133-144.

Benedetti F, Pollo A, Colloca L (2007) Opioid-mediated placebo responses boost pain endurance and physical performance: is it doping in sport competitions? J Neurosci 27:11934-11939.

Bingel U, Lorenz J, Schoell E, Weiller C, Büchel C (2006) Mechanisms of placebo analgesia: $\mathrm{rACC}$ recruitment of a subcortical antinociceptive network. Pain 120:8-15.

Bingel U, Wanigasekera V, Wiech K, Ni Mhuircheartaigh R, Lee MC, Ploner M, Tracey I (2011) The effect of treatment expectation on drug efficacy: imaging the analgesic benefit of the opioid remifentanil. Sci Transl Med 3:70ra14

Bjørkedal E, Flaten MA (2011) Interaction between expectancies and drug effects: an experimental investigation of placebo analgesia with caffeine as an active placebo. Psychopharmacology 215:537-548.

Bush G, Luu P, Posner MI (2000) Cognitive and emotional influences in anterior cingulate cortex. Trends Cogn Sci 4:215-222.

Butler C, Steptoe A (1986) Placebo responses: an experimental study of psychophysiological processes in asthmatic volunteers. Br J Clin Psychol 25:173-183.

Clark WC (1969) Sensory-decision theory analysis of the placebo effect on the criterion for pain and thermal sensitivity. J Abnorm Psychol 74:363-371.

Coghill RC, Sang CN, Maisog JM, Iadarola MJ (1999) Pain intensity processing within the human brain: a bilateral, distributed mechanism. J Neurophysiol 82:1934-1943.

Colloca L, Benedetti F (2005) Placebos and painkillers: is mind as real as matter? Nat Rev Neurosci 6:545-552.

Colloca L, Benedetti F (2006) How prior experience shapes placebo analgesia. Pain 124:126-133.

Colloca L, Benedetti F (2007) Nocebo hyperalgesia: how anxiety is turned into pain. Curr Opin Anaesthesiol 20:435-439.

Colloca L, Benedetti F (2009) Placebo analgesia induced by social observational learning. Pain 144:28-34.

Colloca L, Miller FG (2011a) How placebo responses are formed: a learning perspective. Philos Trans R Soc Lond B Biol Sci 366:1859-1869.

Colloca L, Miller FG (2011b) Harnessing the placebo effect: the need for translational research. Philos Trans R Soc Lond B Biol Sci 366:1922-1930. 
Colloca L, Lopiano L, Lanotte M, Benedetti F (2004) Overt versus covert treatment for pain, anxiety, and Parkinson's disease. Lancet Neurol 3:679-684.

Colloca L, Sigaudo M, Benedetti F (2008a) The role of learning in nocebo and placebo effects. Pain 136:211-218.

Colloca L, Tinazzi M, Recchia S, Le Pera D, Fiaschi A, Benedetti F, Valeriani M (2008b) Learning potentiates neurophysiological and behavioral placebo analgesic responses. Pain 139:306-314.

Colloca L, Petrovic P, Wager TD, Ingvar M, Benedetti F (2010) How the number of learning trials affects placebo and nocebo responses. Pain 151:430-439.

Domnick C, Lorenz J, Hauck M (2011) Somatotopy of placebo analgesia is independent of spatial attention. J Pain Res 4:79-83.

Eippert F, Finsterbusch J, Bingel U, Büchel C (2009a) Direct evidence for spinal cord involvement in placebo analgesia. Science 326:404.

Eippert F, Bingel U, Schoell ED, Yacubian J, Klinger R, Lorenz J, Büchel C (2009b) Activation of the opioidergic descending pain control system underlies placebo analgesia. Neuron 63:533-543.

Feather BW, Chapman CR, Fisher SB (1972) The effect of a placebo on the perception of painful radiant heat stimuli. Psychosom Med 34:290-294.

Flaten MA (2009) Drug effects: agonistic and antagonistic processes. Scand J Psychol 50:652-659.

Flaten MA, Blumenthal TD (1999) Caffeine-associated stimuli elicit conditioned responses: an experimental model of the placebo effect. Psychopharmacology (Berl) 145:105-112.

Flaten MA, Simonsen T, Olsen H (1999) Drug-related information generates placebo and nocebo responses that modify the drug response. Psychosom Med 61:250-255.

Flaten MA, Aslaksen PM, Finset A, Simonsen T, Johansen O (2006) Cognitive and emotional factors in placebo analgesia. J Psychosom Res 61:81-89.

Flaten MA, Aslaksen PM, Lyby PS, Bjørkedal E (2011) The relation of emotions to placebo responses. Philos Trans R Soc Lond B Biol Sci 366:1818-1827.

Garcia-Larrea L, Frot M, Valeriani M (2003) Brain generators of laserevoked potentials: from dipoles to functional significance. Neurophysiol Clin 33:279-292.

Geers AL, Helfer SG, Kosbab K, Weiland PE, Landry SJ (2005) Reconsidering the role of personality in placebo effects: dispositional optimism, situational expectations, and the placebo response. J Psychosom Res 58:121-127.

Geers AL, Wellman JA, Fowler SL, Helfer SG, France CR (2010) Dispositional optimism predicts placebo analgesia. J Pain 11:1165-1171.

Goebel MU, Trebst AE, Steiner J, Xie YF, Exton MS, Frede S, Canbay AE, Michel MC, Heemann U, Schedlowski M (2002) Behavioral conditioning of immunosuppression is possible in humans. FASEB J 16:1869-1873.

Goffaux P, Redmond WJ, Rainville P, Marchand S (2007) Descending analgesia - when the spine echoes what the brain expects. Pain 130:137-143.

Isenberg SA, Lehrer PM, Hochron S (1992) The effects of suggestion and emotional arousal on pulmonary function in asthma: a review and a hypothesis regarding vagal mediation. Psychosom Med 54:192-216.

Jänig W (2006) The integrative action of the autonomic nervous system. Neurobiology of homeostasis. Cambridge, UK: Cambridge UP.

Johansen O, Brox J, Flaten MA (2003) Placebo and nocebo responses, cortisol, and circulating beta-endorphin. Psychosom Med 65:786-790.

Kemeny ME, Rosenwasser LJ, Panettieri RA, Rose RM, Berg-Smith SM, Kline JN (2007) Placebo response in asthma: a robust and objective phenomenon. J Allergy Clin Immunol 119:1375-1381.

Kober H, Barrett LF, Joseph J, Bliss-Moreau E, Lindquist K, Wager TD (2008) Functional grouping and cortical-subcortical interactions in emotion: a meta-analysis of neuroimaging studies. Neuroimage 42:998-1031.

Kong J, Gollub RL, Rosman IS, Webb JM, Vangel MG, Kirsch I, Kaptchuk TJ (2006) Brain activity associated with expectancy-enhanced placebo analgesia as measured by functional magnetic resonance imaging. J Neurosci 26:381-388.

Krummenacher P, Candia V, Folkers G, Schedlowski M, Schönbächler G (2010) Prefrontal cortex modulates placebo analgesia. Pain 148: 368-374.

Lane RD, Wager TD (2009) The new field of brain-body medicine: what have we learned and where are we headed? Neuroimage 47:1135-1140.

Levine JD, Gordon NC, Fields HL (1978) The mechanism of placebo analgesia. Lancet 2:654-657.
Levine JD, Gordon NC, Bornstein JC, Fields HL (1979) Role of pain in placebo analgesia. Proc Natl Acad Sci U S A 76:3528-3531.

Lui F, Colloca L, Duzzi D, Anchisi D, Benedetti F, Porro CA (2010) Neural bases of conditioned placebo analgesia. Pain 151:816-824.

Lyby PS, Aslaksen PM, Flaten MA (2010) Is fear of pain related to placebo analgesia? J Psychosom Res 68:369-377.

Lyby PS, Aslaksen PM, Flaten MA (2011) Variability in placebo analgesia and the role of fear of pain-an ERP study. Pain 152:2405-2412.

Mackenbach JP (2005) On the survival of the altruistic trait in medicine: is there a link with the placebo effect? J Clin Epidemiol 58:433-435.

Matre D, Casey KL, Knardahl S (2006) Placebo-induced changes in spinal cord pain processing. J Neurosci 26:559-563.

Meissner K (2000) Gibt es organspezifische Placeboeffekte? Placeboeffekte an physiologischen Parametern und ihre mögliche Steuerung über kortikale Organrepräsentationen. Aachen, Germany: Shaker.

Meissner K (2009) Effects of placebo interventions on gastric motility and general autonomic activity. J Psychosom Res 66:391-398.

Meissner K (2011) The placebo effect and the autonomic nervous system: evidence for an intimate relationship. Philos Trans R Soc Lond B Biol Sci 366:1808-1817.

Meissner K, Ziep D (2011) Organ-specificity of placebo effects on blood pressure. Autonomic Neuroscience 164:62-66.

Meissner K, Distel H, Mitzdorf U (2007) Evidence for placebo effects on physical but not on biochemical outcome parameters: a review of clinical trials. BMC Med 5:3.

Mikalsen A, Bertelsen B, Flaten MA (2001) Effects of caffeine, caffeineassociated stimuli, and caffeine-related information on physiological and psychological arousal. Psychopharmacology (Berl) 157:373-380.

Montgomery G, Kirsch I (1996) Mechanisms of placebo pain reduction: an empirical investigation. Psychol Sci 7:174-176.

Morrison SF (2001) Differential control of sympathetic outflow. Am J Physiol Regul Integr Comp Physiol 281:R683-R698.

Morton DL, El-Deredy W, Watson A, Jones AKP (2010a) Placebo analgesia as a case of a cognitive style driven by prior expectation. Brain Res 1359:137-141.

Morton DL, Brown CA, Watson A, El-Deredy W, Jones AKP (2010b) Cognitive changes as a result of a single exposure to placebo. Neuropsychologia 48:1958-1964.

Mouraux A, Diukova A, Lee MC, Wise RG, Iannetti GD (2011) A multisensory investigation of the functional significance of the "pain matrix." Neuroimage 54:2237-2249.

Pacheco-López G, Riether C, Doenlen R, Engler H, Niemi MB, Engler A, Kavelaars A, Heijnen CJ, Schedlowski M (2009) Calcineurin inhibition in splenocytes induced by Pavlovian conditioning. FASEB J 23:1161-1167.

Petrovic P, Kalso E, Petersson KM, Ingvar M (2002) Placebo and opioid analgesia — imaging a shared neuronal network. Science 295:1737-1740.

Poldrack RA (2006) Can cognitive processes be inferred from neuroimaging data? Trends Cogn Sci 10:59-63.

Price DD, Craggs J, Verne GN, Perlstein WM, Robinson ME (2007) Placebo analgesia is accompanied by large reductions in pain-related brain activity in irritable bowel syndrome patients. Pain 127:63-72.

Rescorla RA (1988) Pavlovian conditioning. It's not what you think it is. Am Psychol 43:151-160.

Rhudy JL, Williams AE, McCabe KM, Russell JL, Maynard LJ (2008) Emotional control of nociceptive reactions (ECON): do affective valence and arousal play a role? Pain 136:250-261.

Ronel J, Mehilli J, Ladwig K-H, Blättler H, Oversohl N, Byrne RA, Bauer A Schneider S, Linde K, Henningsen P, Lahmann C, Noll-Hussong M, Meissner K (2011) Effects of verbal suggestion on coronary arteries: results of a randomized controlled experimental investigation during coronary angiography. Am Heart J 162:507-511.

Scott DJ, Stohler CS, Egnatuk CM, Wang H, Koeppe RA, Zubieta JK (2008) Placebo and nocebo effects are defined by opposite opioid and dopaminergic responses. Arch Gen Psychiatry 65:220-231.

Shackman AJ, Salomons TV, Slagter HA, Fox AS, Winter JJ, Davidson RJ (2011) The integration of negative affect, pain and cognitive control in the cingulate cortex. Nat Rev Neurosci 12:154-167.

Siegel S (2008) Learning and the wisdom of the body. Learn Behav $36: 242-252$

Tracey I (2010) Getting the pain you expect: mechanisms of placebo, nocebo and reappraisal effects in humans. Nat Med 16:1277-1283. 
Vase L, Robinson ME, Verne GN, Price DD (2003) The contributions of suggestion, desire, and expectation to placebo effects in irritable bowel syndrome patients. An empirical investigation. Pain 105:17-25.

Vase L, Robinson ME, Verne GN, Price DD (2005) Increased placebo analgesia over time in irritable bowel syndrome (IBS) patients is associated with desire and expectation but not endogenous opioid mechanisms. Pain 115:338-347.

Vits S, Cesko E, Enck P, Hillen U, Schadendorf D, Schedlowski M (2011) Behavioural conditioning as the mediator of placebo responses in the immune system. Philos Trans R Soc Lond B Biol Sci 366:1799-1807.

Wager TD, Fields H (2011) Placebo analgesia. In: Wall and Melzack's textbook of pain, Ed 6 (McMahon SB, Koltzenburg M, eds). Oxford: Churchill Livingstone. In press.

Wager TD, Smith EE (2003) Neuroimaging studies of working memory: a meta-analysis. Cogn Affect Behav Neurosci 3:255-274.

Wager TD, Rilling JK, Smith EE, Sokolik A, Casey KL, Davidson RJ, Kosslyn SM, Rose RM, Cohen JD (2004) Placebo-induced changes in fMRI in the anticipation and experience of pain. Science 303:1162-1167.

Wager TD, Scott DJ, Zubieta JK (2007) Placebo effects on human mu- opioid activity during pain. Proc Natl Acad Sci U S A 104:1105611061.

Wager TD, Davidson ML, Hughes BL, Lindquist MA, Ochsner KN (2008) Prefrontal-subcortical pathways mediating successful emotion regulation. Neuron 59:1037-1050.

Wager TD, Atlas LY, Leotti LA, Rilling JK (2011) Predicting individual differences in placebo analgesia: contributions of brain activity during anticipation and pain experience. J Neurosci 31:439-452.

Watson A, El-Deredy W, Iannetti GD, Lloyd D, Tracey I, Vogt BA, Nadeau V, Jones AKP (2009) Placebo conditioning and placebo analgesia modulate a common brain network during pain anticipation and perception. Pain 145:24-30.

Wechsler ME, Kelley JM, Boyd IO, Dutile S, Marigowda G, Kirsch I, Israel E, Kaptchuk TJ (2011) Active albuterol or placebo, sham acupuncture, or no intervention in asthma. N Engl J Med 365:119-126.

Zubieta JK, Bueller JA, Jackson LR, Scott DJ, Xu Y, Koeppe RA, Nichols TE, Stohler CS (2005) Placebo effects mediated by endogenous opioid activity on mu-opioid receptors. J Neurosci 25:7754-7762. 